Assessing the prospects for achieving double-shell ignition on the National Ignition Facility using vacuum hohlraums

P. Amendt, C. Cerjan, A. Hamza, D. Hinkel, J. L. Milovich , H. F. Robey

November 3, 2006

Physics of Plasmas 
This document was prepared as an account of work sponsored by an agency of the United States Government. Neither the United States Government nor the University of California nor any of their employees, makes any warranty, express or implied, or assumes any legal liability or responsibility for the accuracy, completeness, or usefulness of any information, apparatus, product, or process disclosed, or represents that its use would not infringe privately owned rights. Reference herein to any specific commercial product, process, or service by trade name, trademark, manufacturer, or otherwise, does not necessarily constitute or imply its endorsement, recommendation, or favoring by the United States Government or the University of California. The views and opinions of authors expressed herein do not necessarily state or reflect those of the United States Government or the University of California, and shall not be used for advertising or product endorsement purposes. 


\title{
Assessing the prospects for achieving double-shell ignition on the National Ignition Facility using vacuum hohlraums
}

\author{
Peter Amendt, C. Cerjan, A. Hamza, D.E. Hinkel, J.L. Milovich, and H.F. Robey \\ Lawrence Livermore National Laboratory, University of California, Livermore CA 94551
}

USA

\begin{abstract}
The goal of demonstrating ignition on the National Ignition Facility (NIF) has motivated a revisit of double-shell (DS) targets as a complementary path to the cryogenic baseline approach. Expected benefits of DS ignition targets include non-cryogenic deuterium-tritium (DT) fuel preparation, minimal hohlraum-plasma mediated laser backscatter, low thresholdignition temperatures $(\approx 4 \mathrm{keV})$ for relaxed hohlraum $\mathrm{x}$-ray flux asymmetry tolerances, and minimal (two-) shock timing requirements. On the other hand, DS ignition presents several formidable challenges, encompassing room-temperature containment of high-pressure DT $(\approx$ $790 \mathrm{~atm})$ in the inner shell, strict concentricity requirements on the two shells $(<3 \mu \mathrm{m})$, development of nano-porous $(<100 \mathrm{~nm})$ low-density $(<100 \mathrm{mg} / \mathrm{cc})$ metallic foams for structural support of the inner shell and hydrodynamic instability mitigation, and effective control of hydrodynamic instabilities on the high-Atwood number interface between the DT fuel and the high-Z inner shell. Recent progress in DS ignition designs and required materials-science advances at the nanoscale are described herein. Two new ignition designs that use rugby-shaped vacuum hohlraums are presented which utilize either $1 \mathrm{MJ}$ or $2 \mathrm{MJ}$ of laser energy at $3 \omega$. The capability of the NIF to generate the requested reverse-ramp pulse
\end{abstract}


shape for DS ignition is expected to be comparable to the planned high-contrast $(\approx 100)$ pulse-shape at 1.8 MJ for the baseline cryogenic target. Nano-crystalline, high-strength, Au$\mathrm{Cu}$ alloy inner shells are under development using electrochemical deposition over a glass mandrel, exhibiting tensile strengths well in excess of 790 atm. Novel, low-density (85 $\mathrm{mg} / \mathrm{cc}$ ) copper foams have recently been demonstrated using $10 \mathrm{mg} / \mathrm{cc} \mathrm{SiO}_{2}$ nano-porous aerogels with suspended $\mathrm{Cu}$ particles. A prototype demonstration of an ignition DS is planned for 2008, incorporating the needed novel nano-materials science developments and the required fabrication tolerances for a realistic ignition attempt after 2010 .

\section{Introduction}

The National Ignition Facility [1] is scheduled for a first ignition attempt in 2010 using the indirect-drive configuration. The mainline target will be a cryogenic single-shell capsule [2] capable of high thermonuclear yield (> 10 MJ). A successful demonstration will require (1) cryogenic fielding of the single-shell target with $<1 \mu \mathrm{m}$ DT ice roughness (RMS), (2) control of plasma-mediated laser backscatter reflectivity to $<15 \%$ levels, (3) careful timing of four shocks to within \pm 100 ps with a high-contrast $(\approx 100: 1)$ laser pulse for low-adiabat compressions, and (4) strict control of time-integrated and time-dependent hohlraum x-ray flux non-uniformity. In meeting these goals, much progress has been demonstrated on former (NOVA [3]) and existing (OMEGA [4]; NIF [5]) laser facilities to support a credible ignition attempt in 2010.

A complementary ignition scheme uses a double-shell target $[6,7]$ that will be fielded at room temperature for simplicity [see Fig. 1]. Other advantages include looser tolerances on (2-) shock 
timing, relaxed requirements on lowest-order $\mathrm{x}$-ray flux symmetry control $(\approx 4 \times)[8]$, and expected lower levels of laser backscatter with the reverse-ramp pulse shape. The mode of double-shell ignition will be volume (" $P d V$ ") instead of "hot spot" as in the cryogenic baseline design. Hot-spot ignition on the NIF requires a threshold fuel temperature of nearly $10 \mathrm{keV}$, whereas volume ignition is predicted to occur at $\approx 4 \mathrm{keV}$ as a result of radiation trapping by the high-Z inner shell. Due to the low threshold-ignition temperature for double shells, added robustness to candidate sources of asymmetry, e.g., x-ray flux and particular classes of fabrication errors, is predicted [8]. With little need for isentropic preparation of the roomtemperature fuel, careful pulse shaping for pre-forming the pusher (as in hot-spot ignition) is unnecessary since the in situ high- $Z$ inner shell reaches the required density $\left(>1000 \mathrm{~g} / \mathrm{cm}^{3}\right)$ after converging by only a factor-of-10. Therefore, the preferred mode of energy delivery to the outer shell (ablator) is nearly impulsive, i.e., use of a low-contrast laser pulse shape to simplify laser operations. In addition, delivering the laser energy as early as possible has the advantage of reaching peak laser power before the hohlraum has had sufficient time to fill with plasma and create the potential for laser backscatter. A further advantage of impulsive energy delivery is that the temporal variations in x-ray flux asymmetry on the capsule are lessened [9], thereby relaxing the need for dynamic beam phasing between the inner and outer laser cones and, in turn, making optimal use of the available driver energy on the NIF.

Double shells have their own set of challenges to overcome. The original justification for dropping double shells as a mainline ignition option was their evident susceptibility to turbulence-induced shell breakup following shell collision [6]. In the early 1980s when this apparent failure mode for double shells was generally recognized, the available simulation tools 
were not up to the task of reliably modeling such a presumably turbulent regime (which is still the case today). Thus, the prudent course was to pursue a target designed to minimize nonlinear growth that may lead to turbulence. The cryogenic single-shell design sidesteps the highly nonlinear regime of instability growth by suppressing high-mode growth with density-gradient stabilization across the fuel/pusher "interface" that separates (central) hot DT fuel from the dense DT pusher. However, it is now realized that the interface between the DT ice and the low- $Z$ ablator is not stabilized (in the absence of ionic mass and momentum transport) for high modenumber perturbations and could potentially provide a venue for appreciable growth [10] - or even the onset of turbulence. Other recognized hurdles with a double-shell path to ignition are explaining a legacy of underperforming experiments on NOVA and OMEGA in the 1990s and the daunting challenge of fabricating ignition-ready double shells. The first hurdle has largely been overcome with a successful double-shell implosion campaign on OMEGA in 2003 that featured novel precision machining and assembly techniques $[11,12]$. Overcoming the second challenge is now within reach based both on continuing advances in fabricating high-quality OMEGA-scale double shells and a host of nanoscale-based materials science advances. The materials science developments were largely dictated by the requirements of a double-shell design meant to mitigate hydrodynamic instability growth on the outside of the inner shell after shell collision [13]. The basic feature of the design is that a materials match between the outside of the inner shell and the supporting inter-shell foam will greatly suppress instability according to high-resolution multimode simulations. With the inner shell consisting of gold on the innermost surface and successive layers of increasing copper concentration to provide needed shell material strength, the contiguous foam must consist of low-density $(<100 \mathrm{mg} / \mathrm{cc})$, nanoporous (cell size $<100 \mathrm{~nm}$ ) copper. Diffusion filling with DT through the inner shell requires 
elevated temperatures $\left(>500^{\circ} \mathrm{C}\right)$ for several hours or more. At room temperature the shell must hold $790 \mathrm{~atm}$ of DT, putting severe constraints on the materials properties of the inner shell: the gold and copper must remain in a nano-crystalline state to maintain sufficient strength. Thus, we conclude that realization of a reasonably stable double-shell ignition design must require progress in (and implementation of) nano-based materials science research.

A credible ignition effort must consist of four essential elements: (1) target design and supporting simulations, including 2- and 3-D integrated hohlraum techniques as well as capsuleonly calculations for assessing hydrodynamic instability; (2) analytical tools and methods for code benchmarking and design advances; (3) experiments for code and design validation; and (4) target manufacturing, including materials science development, target fabrication, precision assembly and metrology. Examples of analytical progress can be found in a recent work [14], while multimode simulations are the subject of former [13] and ongoing work. The status of double-shell experiments on OMEGA is summarized elsewhere [11]. This paper will focus on recent advances in hohlraum design for high-gain double-shell performance and highlights of recent nano-based materials science research that offers the prospect of delivering a prototype double-shell ignition target in 2008. The principal design advances are the first vacuumhohlraum ignition design for the NIF and the use of a rugby-like hohlraum geometry. Aside from simplicity in fielding, a vacuum-hohlraum design offers the potential for minimal laser backscatter as low-Z liners and gas fills for auxiliary flux symmetry control are pointedly avoided. The introduction of rugby hohlraums is a further advance in increasing the coupling of laser energy to capsule absorbed energy. More absorbed x-ray drive energy translates into added 
margin to hohlraum flux asymmetry, instability-induced atomic mix between the fuel and inner shell, and target fabrication errors.

This paper is organized as follows. In Section 2 we describe vacuum, rugby hohlraum designs

that use 2 MJ of $3 \omega$ energy with an emphasis on x-ray drive and asymmetry issues. Section 3 assesses the linear backscatter gain exponents for stimulated Brillouin and Raman laser backscatter in vacuum, rugby hohlraums driven by $2 \mathrm{MJ}$ of laser energy. Section 4 considers a candidate $1 \mathrm{MJ} 3 \omega$ design based as well on a vacuum, rugby hohlraum. In Section 5 we highlight some of the developments in nano-scale materials science that pertain to the enablement of an ignition prototype demonstration in 2008. We summarize our results in Section 6.

\section{High-gain double-shell design with vacuum hohlraums}

Non-cryogenic double-shell ignition results in less thermonuclear gain than the mainline cryogenic single-shell design owing simply to significantly less fuel mass, e.g., $\approx 6 \times$ for $1 \mathrm{MJ}$ at $3 \omega$ designs. To improve the yield of non-cryogenic double shells requires innovative approaches to coupling more hohlraum-generated x-ray energy to the outer-shell ablator. For a given cylindrical hohlraum case radius, the case-to-capsule ratio (CCR) can be decreased to increase the capsule absorbed energy - but at the obvious expense of likely aggravating the x-ray drive asymmetry. For example, increasing the outer-shell radius will lead to further shadowing of the hohlraum wall near the equator, resulting in locally lower drive over a significantly large solid angle and a stubborn source of flux asymmetry on target. Rather, another option is to modify the geometry of the hohlraum from a cylinder to a rugby shape, providing more volume over the 
equator as needed. Previous ignition designs, both for double shells [6] and single shells [15], have included gas fills or low- $Z$ liners to control hohlraum flux asymmetry by suppressing the radial inward expansion of the (cylindrical) hohlraum wall. A feature of these hohlraum designs is the risk of laser backscatter, potentially resulting in lower x-ray drive and yet another source of drive asymmetry. Although use of the reverse-ramp pulse shape implies a reduced risk of laser backscatter for double-shell targets, a helium-filled hohlraum does still require complicating windows and likely cryogenic cooling for gas confinement.

To simplify target fielding and increase the prospects for higher-gain double-shell ignition, we focus on vacuum hohlraum designs for the NIF. A further design development is the replacement of the high-Z cylindrical hohlraum with a rugby-shaped enclosure [See Fig. 2(a)]. There are several advantages to using a rugby-shaped hohlraum compared with a cylinder of the same surface area. First and foremost, more available volume above the outer-shell equator (or waist) means less shadowing of the hohlraum wall by the double shell. Therefore, a larger double shell can be fielded without incurring an excessive flux deficit near the waist of the outer shell. With the comparatively large solid angle above the waist of the outer shell, there is more leverage for enhancing the average drive on target. Second, a larger hohlraum radius on the equator enables more vacuum transport smoothing of radiation asymmetries incident on the outer shell. The degree of radiation smoothing from wall to capsule is generally a strong function of "case-tocapsule" ratio [16], particularly for higher mode asymmetries. With the greater proximity of the hohlraum waist compared with the corner of a cylinder, the effective case-to-capsule ratio of the rugby target intuitively should be greater based on a view-factor $\left(\propto 1 / r_{12}^{2}\right.$, where $r_{12}$ is the distance between differential surface area elements 1 and 2) assessment (e.g., [17]). Thus, some 
improvement in hohlraum smoothing of radiation asymmetry between the wall and outer shell is expected with a rugby hohlraum compared with a cylinder of the same surface area. Third, specular reflection of laser light onto the outer shell will be less due to the innate curvature of the rugby shape and the relatively low angles of incidence in the outer cones. The inner pair of cones $\left(23,30^{\circ}\right)$ that intercept the rugby hohlraum wall past the waist are expected to have relatively higher conversion efficiency at early time because of their high angle of incidence. A fourth potential advantage of a rugby-like hohlraum geometry is reduced lowest-order time-dependent symmetry swings. In a multicone geometry, previous analysis has shown that placement of the inner cones to intercept a cylindrical hohlraum wall near the waist (or node of the second Legendre polynomial) and the outer cones near the symmetry axis has the benefit of reducing the amplitude of lowest-order, time-dependent, flux asymmetry swings from laser spot motion [18]. In the case of a rugby-shaped hohlraum the outer cones intercept the wall relatively close to the axis due to the curvature of the hohlraum. A final potential benefit of the rugby geometry is that a more spherical-like shape compared with a cylinder ensures that the Legendre polynomials are a truer representation of the eigenfunctions of vacuum radiation transport. In this way, less mode coupling is predicted which helps simplify the strategies for hohlraum flux symmetry control. Based on these five supporting arguments for adopting a rugby design, we have focused our ignition tuning efforts (with 2-D integrated hohlraum simulations [9]) on this geometry. Further work to quantify the energetics and viewfactor properties of rugby hohlraums in comparison with cylinders is planned shortly. 
Figure 2(b) illustrates how the rugby-shaped hohlraum is geometrically constructed. A crosssection or azimuthal cut of the rugby consists of two circular arcs whose origins are separated by $2 R_{0}$ :

$$
R_{0}=\frac{z_{h}^{2}+\left(R_{h}-R_{l e h}\right)^{2}}{2\left(R_{h}-R_{l e h}\right)}
$$

where $z_{h}$ is the (half) hohlraum length, $R_{h}$ is the hohlraum radius at the waist $(z=0)$ and $R_{\text {leh }}$ is the radius of the laser-entrance-hole (LEH) opening. The surface area of the rugby $A$ is readily obtained:

$$
A=4 \pi R_{0}\left[z_{h}-\left(R_{0}-R_{h}\right) \sin ^{-1}\left(\frac{z_{h}}{R_{0}}\right)\right] .
$$

Figure 3 shows a comparison between rugby and cylindrical hohlraums (of the same wall surface area $A$ ) for the time-integrated (normalized) $P_{2}$ outer-shell ablation pressure up to the time of shell collision versus scaled outer-shell radius. The wall material consists of $75 / 25 \% \mathrm{U} / \mathrm{Au}$ cocktail mixture for enhanced target coupling [19]. Compared with a cylinder, the sensitivity of lowest-order flux asymmetry $\left(\mathrm{P}_{2}\right)$ with increasing outer-shell radius for a rugby shape is more than a factor-of-two lower. This trend is expected as a result of the smaller $R_{h}(0.867 \times)$ for cylinders and the increasing effect of shadowing of the wall as the outer-shell size is increased. Thus, a rugby-hohlraum geometry offers the prospect of realizing larger double-shell target designs with more thermonuclear yield. Larger-sized double shells are also of interest from the standpoint of margin to deleterious flux asymmetry, target fabrication errors and susceptibility to mix. For example, a fall-line analysis applied to mixing of high- $Z$ pusher and DT fuel confirms the benefit of accessing higher absorbed x-ray energies. Recall that the fall line is the straightline trajectory of interfacial material at deceleration onset (or peak implosion speed). The notion 
is that no pusher material is expected ahead of the fall-line trajectory based on causality, thus placing an upper limit on the extent of mixing. Thus, comparing fall-line trajectories relative to the onset of thermonuclear burn can provide a handy metric for comparing and assessing the margins to mix. In the case of a $1 \mathrm{MJ}$ design (See Sec. 5) that absorbs nearly $500 \mathrm{~kJ}$ in $1 \mathrm{D}$, the fall line reaches the origin $(r=0)$ about 25 ps before peak burn. On the other hand, the $2 \mathrm{MJ}$ design that absorbs nearly $850 \mathrm{~kJ}$ in $1 \mathrm{D}$ has a fall line that follows the time of peak burn by more than 40 ps ( $\approx$ FWHM burn width). Reliably gauging the degree of mix in an ICF implosion is always a formidable challenge, and any means to minimize its effects serves to enhance overall target robustness.

Figure 4 shows the proposed laser power history and a constant cone-to-cone power fraction, i.e., no dynamic beam phasing between the inner and outer beams for auxiliary symmetry control is used. Such a power history makes optimal use of the NIF laser architecture for the requested 2 MJ of $3 \omega(0.351 \mu \mathrm{m})$ energy. The beam-phasing option is expendable because symmetry control can be adequately provided by use of a sufficiently short pulse [9]. Figure 5 shows the $2^{\text {nd }}$ and $4^{\text {th }}$ Legendre coefficients of the x-ray flux incident on the outer shell as predicted by $2 \mathrm{D}$ integrated hohlraum simulations. Between 1 and 4 ns over which time the hohlraum drive is maximized and nearly constant $(\approx 260 \mathrm{eV})$, the lowest-order $\left(\mathrm{P}_{2}\right)$ symmetry variations are $2 \%$ or less. The highorder flux asymmetry remains at manageably low levels. The flux asymmetry coefficients are then used in a capsule-only simulation to assess implosion performance. The resulting yield is $\approx 9$ MJ, close to the 1D yield of 10 MJ. Such a high value of gain has not been achieved with standard cylindrical hohlraums to date, presumably due to intolerably high time-integrated flux asymmetry $\left(\mathrm{P}_{2}>0\right)$ and significant flux asymmetry swings. The corresponding capsule design is 
energetically very efficient, absorbing nearly $0.85 \mathrm{MJ}$ in $1 \mathrm{D}$ simulations that use a frequencydependent-source extracted from an integrated hohlraum simulation. This amount of absorbed energy compares favorably with a high-gain cryogenic double-shell design [6] that is calculated to generate a $1 \mathrm{D}$ yield of $313 \mathrm{MJ}$ while absorbing $0.80 \mathrm{MJ}$.

\section{Laser backscatter for the $2 \mathrm{MJ}$ design}

The Laser Interaction with Plasma post-processor [20] was used to assess plasma-mediated laser backscatter in the rugby vacuum-hohlraum design. LIP uses the simulated flow speeds, temperatures, densities and materials properties along a (straight) laser ray path to estimate the steady state, kinetic, linear growth factors for stimulated Brillouin backscatter (SBS) and stimulated Raman backscatter (SRS). Figure 6(a) shows the simulated peak Raman and Brillouin backscatter gain-length exponents for the four NIF cones and $1.5 \mathrm{~mm}$ diameter focal spots. The peak SRS gain lengths are quite benign $(<3)$, while SBS is only modestly high $(<19)$. Note that the peak SBS gain occurs nearly $1.5 \mathrm{~ns}$ after peak power and is rather transient, lasting less than 300 ps. A more meaningful gain-exponent figure-of-merit is a power-weighted average by which the transient bursts are temporally smoothed and the gains near peak power are highly weighted; for the case at hand we find a weighted gain exponent $\langle G L>\approx 14$. Whichever convention or metric is adopted for representing linear backscatter signal gain, a direct correspondence with a reflectivity coefficient (measured or predicted) must be made for the ultimate guidance on defining a threshold value of tolerable gain. We will argue shortly that a "peak" gain exponent of $\approx 10$ corresponds to a tolerable reflectivity of a few percent, based on a scaling comparison of the

2 MJ design with a recent Omega experiment that had well-characterized backscatter levels. To 
achieve such a lower gain exponent, a decrease in the beam focal spot intensity by nearly $2 \times$ is sought. In such a case (of a $2.25 \mathrm{~mm}$ diameter focal spot) the predicted SBS gain lengths are all less than 10, while the SRS gain lengths are uniformly less than 1. A set of 192 phase plates that can accommodate such large focal spots on the NIF may not be readily available, as smaller phase plates are currently planned for the baseline single-shell ignition campaign. However, a recently reported technique [21] that combines defocusing and repointing of the 4 beams within a quad may provide the needed flexibility of larger focal spots for double-shell experiments.

A useful exercise for relating calculated backscatter gain exponents with a reflectivity is to analytically compare SRS and SBS growth-rate scalings for our $2 \mathrm{MJ}$ design with the extensive vacuum-hohlraum database on the NOVA and OMEGA laser facilities. Typically, vacuumhohlraum experiments on these facilities have resulted in relatively low backscatter reflectivities compared with gas-filled hohlraums, provided that the targets are of the normal type or dimension ("scale-1"). Only recently has the OMEGA laser been fitted with phase plates in the indirect-drive configuration, which allows for a more relevant comparison with simulated ignition-hohlraum conditions. The OMEGA experiment used a $1 \mathrm{~ns}$ flattop laser pulse of $20 \mathrm{~kJ}$ distributed over 40 beams, compared with $2 \mathrm{MJ}$ that is delivered over $\approx 3 \mathrm{~ns}$ (with a 2 ns rise time) and distributed among 48 quads of the NIF. The cylindrical scale-1 vacuum hohlraum volume was $\approx 0.005 \mathrm{~cm}^{3}$, or about $s=110$ times smaller than our $2 \mathrm{MJ}$ rugby design. Thus, the hohlraum energy densities between OMEGA and the NIF design are within 10\%. The measured peak Dante [22] hohlraum temperature was $\approx 230 \mathrm{eV}$, compared with a peak value of $\approx 260 \mathrm{eV}$ in our simulated NIF ignition design. The OMEGA experiment was fielded without a capsule, though the $66 \%$ LEHs are significantly larger than the $41.7 \%$ LEHs in our ignition design. The 
peak beam intensity $I$ in the experiment was $\approx 1.1 \times 10^{15} \mathrm{~W} / \mathrm{cm}^{2}$ compared with a peak (quad) intensity of $5 \times 10^{14} \mathrm{~W} / \mathrm{cm}^{2}$ in our design that uses $2.25 \mathrm{~mm}$ diameter focal spots; the NIF beam path lengths $L$ are about 3-4.5 times larger than on OMEGA, depending on the particular beam cone. The amount of hohlraum filling (by the end of the pulse) can be defined as the areal massablation rate of the high-Z wall ( $d m / d t \propto T_{R}^{1.86} \tau^{-0.46}$ for constant $T_{R}$ [2]) times the pulse length $\tau$ times the hohlraum surface area $A$, where $T_{R}$ is the hohlraum radiation temperature. Thus, the mass per unit volume $\left(\propto n_{e}\right)$ ablated in the NIF hohlraum is about one-half of the OMEGA case, using that $A \approx s^{2 / 3}$. With the energy density $\left(\propto n_{e} T_{e}\right) 10 \%$ higher for the OMEGA experiment, it also follows that $T_{e}$ should be nearly $1.9 \times$ greater in the NIF design. Finally, using the scaling relation for both SRS and SBS gain exponents $\propto I \cdot L \cdot n_{e} / T_{e}$, we estimate that the NIF linear SRS and SBS gain exponents are within a factor-of-2 of the OMEGA experiment which has an inferred total backscatter reflectivity of only $\approx 4 \%$ [23]. Obviously, this comparison has its limits, e.g., differences in pulse shape, absence of an OMEGA capsule, cylinder versus rugby, and different beam angles, but the exercise portends favorably for our NIF double-shell ignition design. LIP calculations of the OMEGA intensity gain exponents $(G L)$ give $\mathrm{SRS}<2$ for all three cones, in good agreement with the predicted NIF SRS gain exponents $(<1)$ for all four cones. The OMEGA SBS gain exponents are also $<10$ for all cones as in our NIF design, though the distribution among the three beam cones on OMEGA [GL $\left.<10\left(22^{\circ}\right), 1\left(42^{\circ}\right), 4\left(59^{\circ}\right)\right]$ is reversed relative to NIF's four cones $\left[G L<4\left(23^{\circ}\right), 7\left(30^{\circ}\right), 7\left(44^{\circ}\right), 10\left(50^{\circ}\right)\right]$. This reversal is presumably due to the outer NIF cones having relative shallow angles of incidence in a rugby hohlraum in contrast to the low incidence-angle inner cones of an OMEGA cylindrical hohlraum. Overall, the predicted LIP gains for the OMEGA experiment and our NIF ignition design are quite comparable despite the differences as noted above. More importantly, we are able to 
benchmark our calculated gain exponents, whether defined as peak values or power weighted values, with experimental reflectivities. Further OMEGA experiments with phase plates and capsules are planned over the near term that should provide more stringent tests of our ability to predict backscatter in proposed NIF ignition hohlraums.

A useful figure-of-merit for gauging beam filamentation and spray is the filamentation threshold $Q$ [24]:

$$
Q=3 I\left[\mathrm{~W} / \mathrm{cm}^{2}\right] \cdot \lambda^{2}[\mu \mathrm{m}] \cdot\left(n_{e} / n_{c}\right) / T_{e}[\mathrm{keV}] \cdot\left(\frac{f}{8}\right)^{2}<10^{13},
$$

where $\lambda=0.351 \mu \mathrm{m}$ is the laser wavelength, $n_{e} / n_{c}$ is the electron density normalized to the critical density at $3 \omega$ and $f$ is the lens $f$-number. Evaluating Eq. (3) for $1.5 \mathrm{~mm}$ focal spots indicates that $Q$ remains well below the filamentation threshold for all cones at all times. The same $n_{e} / T_{e}$ factor occurs in the linear growth-rate expressions for SRS and SBS and is partly responsible for the predicted modest gain exponents. At peak power the electron density is less than $3 \%$ of critical over $90 \%$ of the beam path length within the LEH, and the electron temperature is $3-5 \mathrm{keV}$. A further contributing factor to the above-mentioned low SRS gains is the presence of strong density gradients. With a short laser pulse $(\approx 3 n s)$ to realize adequate vacuum-hohlraum symmetry control, the hohlraum has not yet filled with plasma to enable formation of density plateaus that can drive SRS. 


\section{1 MJ 3w design}

The initial ignition campaigns to be performed on NIF in 2010 will use a single-shell point design with the required laser energy of 1MJ. Subsequent experiments will move to higher laser energy as necessary to mitigate performance risk in the target design. A lower energy doubleshell ignition design is of interest from the standpoints of ignition readiness and ease of laser operations, as well as gauging the predicted decline in performance margin with less absorbed energy. Aside from a $2^{-1 / 3}$ overall scale-factor compared with the $2 \mathrm{MJ}$ design (See Section 2), an added simulation feature is the inclusion of an inner Au liner $(0.2 \mu \mathrm{m})$ to protect the U/Au cocktail wall from oxidation. To accommodate the drive loss (of several $\mathrm{eV}$ in radiation temperature) resulting from the presence of the protective gold layer, the overall scale-factor of the rugby geometry is reduced by an additional $11 \%$ (to 0.70 ) relative to the $2 \mathrm{MJ}$ hohlraum design without the gold liner (Section 2). The peak hohlraum temperature is nearly $270 \mathrm{eV}$ and the maximum laser power is $345 \mathrm{TW}$. The $1 \mathrm{D}$ target yield is $3.65 \mathrm{MJ}$ and the fall line advance relative to the instant of peak burn is $\approx+25$ ps. Two-dimensional capsule-only simulations with imposed time-dependent $\mathrm{x}$-ray flux asymmetries from an integrated hohlraum calculation give a yield of $\approx 2.5 \mathrm{MJ}$, or $70 \%$ of the $1 \mathrm{D}$ yield. The capsule absorbed energy in $1 \mathrm{D}$ simulations is nearly $520 \mathrm{~kJ}$. The high coupling is a reflection of the relatively small rugby hohlraum compared with the $2 \mathrm{MJ}$ design (See Section 2), the large outer shell and the high x-ray albedo associated with the reverse-ramp pulse shape (See Fig. 4).

Figure 7 shows the double-shell configuration at the instant of peak thermonuclear burn according to a well-resolved multi-mode Hydra simulation [25]. The calculation includes 
Legendre modes from $\ell=12-408$ with 20 angular zones per smallest wavelength. Despite the modestly unfavorable fall-line behavior for this target, the degree of instability growth on the fuel-pusher interface is not large and the inner shell remains well intact. The target design strove for as small a shell aspect ratio $(\approx 7)$ as possible for margin against perturbation feed-through (from the outside surface) and potential shell breakup. The roughness spectrum used in the calculation was borrowed from a measured glass spectrum with an RMS roughness of $30 \mathrm{~nm}$ for mode numbers in the range 12-408. The inner third of the outer shell used double the amount of $\mathrm{Cu}$ dopant compared with the $2 \mathrm{MJ}$ design for higher peak implosion speeds and improved fallline behavior. The yield from this calculation was $\approx 2 \mathrm{MJ}$, or nearly $60 \%$ of the $1 \mathrm{D}$ yield.

Figure 8 displays the peak SRS and SBS gain-length products for each cone. Raman backscatter is of little apparent concern, remaining below 3 gain lengths. Brillouin backscatter is a potential concern with the $30^{\circ}$ cone, but the peak $(G L)$ value $\approx 20$ occurs after $2 \mathrm{~ns}$, well after the time of peak laser power (1 ns) and is also rather transient, lasting only 200 ps or less. In a powerweighted sense the SFS gain exponent for the $30^{\circ}$ cone will be close to 10 as required, based on scaling arguments that were applied to a well-characterized OMEGA experiment [See Section 3]. The phase plates used in this design provide focal spots of $1.3 \mathrm{~mm}$ diameter for all the beams.

Overall, predicted control of flux asymmetry, hydrodynamic instability and laser backscatter portend favorably for an ignition demonstration, despite the relatively low thermonuclear gain. Further simulation work to combine hohlraum flux asymmetry with hydrodynamic growth to assess Kelvin-Helmoltz instability growth is planned. 


\section{Materials science advances}

The ignition double shells depicted in Fig. 1 require several nano-based materials science advances to enable a prototype demonstration. First, the bimetallic inner shell must be graded from pure $\mathrm{Au}$ at the inner edge to pure $\mathrm{Cu}$ on the outside and have sufficient material strength to contain 790 atm of DT fill at room temperature. By the well-known Hall-Petch effect [26], material strength is known to increase with decreasing grain size down to a threshold of $\approx 10-20$ nm; yet smaller grain sizes are known to lead to reduced strength. The technique of choice for manufacturing high-strength inner shells is electrochemical deposition that can provide the required nano-crystalline grain structure.

Figure 9 shows a transmission electron micrograph of a planar sample of $\mathrm{Au}-\mathrm{Cu}$ alloy with a characteristic $20 \mathrm{~nm}$ grain size. Pull tests have verified that the samples are as strong as expected, exhibiting more than $600 \mathrm{MPa}$ [592 atm] of tensile strength or about six times larger than for coarse-grained $\mathrm{Au}-\mathrm{Cu}$. Recently, the same process has been successfully demonstrated for spheres by electrodepositing $\mathrm{Au}$ and $\mathrm{Cu}$ over a glass mandrel. Figure 10 shows some samples of $\mathrm{Au}-\mathrm{Cu}$ shells where the inner $5 \mu \mathrm{m}$ consists of pure Au over-coated with $5 \mu \mathrm{m}$ of pure $\mathrm{Cu}$. The inner mandrel is removable by laser drilling through the shell and then chemically leaching with an acid solution in an ultrasonic bath. Hole plugging is planned using focused-ion-beam chemical vapor deposition. Addition of "pinning" agents for suppressing grain growth as the shell is subjected to elevated temperatures $\left(>500^{\circ} \mathrm{C}\right)$ during diffusion filling with DT will be needed. Tests on Au-Cu flats with 5 wt.\% Sn have indeed established that high tensile strength is preserved ( $>570 \mathrm{MPa})$ while maintaining good ductility ( $\approx 5 \%$ strain). 
Another required nano-science advance for the prototype double shell is the development of nanoporous, low-density, metallic foams. The current ignition design (See Fig. 1) calls for a copper foam between the two shells to mitigate hydrodynamic instability on the outside of the inner shell following shell collision [13]. Recently, a sub-100 mg/cc sample was fabricated using aerogel gluing. In this method copper particles in solution compatible with aerogel processing are mixed with a silica sol-gel precursor. The precursor is then allowed to gel. A uniform gel is then transformed to an aerogel by supercritical extraction of the "glue". The $\mathrm{Cu}$ particles were approximately $1 \mu \mathrm{m}$ in diameter and unevenly dispersed in the silica aerogel with separations up to $5 \mu \mathrm{m}$ [See Fig. 11a]. The resulting foam density was $85 \mathrm{mg} / \mathrm{cc}$ with 12 wt.\% of $\mathrm{SiO}_{2}$ aerogel. Figure 12 shows the results of $1 \mathrm{D}$ simulations to assess the effect of impurities in the nominal copper foam on the Atwood number (or density-mismatch parameter) of the inner-shell/foam interface. Because dangerous Rayleigh-Taylor hydrodynamic instability growth rates are proportional to the square root of the Atwood number, any significant reduction of this parameter can help suppress such growth. The double-shell ignition target designs (See Fig. 1) are intended to engineer a vanishing Atwood number on this interface by imposing an exact material match. In practice, this matching will not be exact and a residually low Atwood number will still allow some instability growth. Figure 12 assesses the impact of residual glass on the copper foam compared with the idealized case of a $\mathrm{Cu} / \mathrm{Cu}$ interface and the worst case of a pure $\mathrm{Cu} / \mathrm{SiO}_{2}$ (aerogel) interface. Clearly, the delivered foam with its silicon impurity is quite close to the idealized case after 8 ns (when the acceleration becomes large) and represents a large improvement over the pure aerogel extreme. Work to achieve a nano-porous $\mathrm{Cu}$ foam by significantly reducing the $\mathrm{Cu}$ particle sizes is continuing. Figure $11 \mathrm{~b}$ shows the results of a newer 
technique with copper-oxide aerogels that now realizes sub-micron pore sizes and a density of 50 $\mathrm{mg} / \mathrm{cc}$. Further work to explore the machinability of these foams as well as pursuing simulation studies on the effect of instability seeding from moderate-sized pore sizes has begun.

A final development is based on the rapid advances in diamond-film technology. Nanocrystalline carbon shells with high density $\left(3.51 \mathrm{~g} / \mathrm{cm}^{3}\right)$, smooth surface finishes and ignitionrelevant dimensions have been demonstrated [See Fig. 13]. The high degree of $\mathrm{sp}^{3}$ molecular purity $(>99.5 \%)$ and relatively small grain sizes $(<1 \mu \mathrm{m})$ provide good reason to consider this material as an outer-shell ablator candidate. An additional application of high-density carbon shells could be as a replacement for the glass inner-shell mandrels. The lower atomic number of carbon (6) compared with silicon (13) would mean more margin to fuel-pusher mixing at ignition - if the mandrel is chosen to remain in place.

\section{Summary}

A high-gain, non-cryogenic, double-shell ignition design is described. The main improvement of this design over previous work $[7,13]$ is the use of vacuum hohlraums. Vacuum hohlraums are simpler to field, i.e., no windows or cryogenic gas fill are needed, and a large database for vacuum cylindrical hohlraum experiments generally shows favorable low levels of plasmainduced laser backscatter. A further design improvement is the use of rugby-shaped hohlraums to avail more drive and symmetry control. Experiments with rugby hohlraums on Omega are planned shortly to test our understanding of hohlraum drive and symmetry in this new geometry. Cylindrical hohlraum designs remain a strong option for a double-shell ignition design mainly 
because of their simplicity in fabrication and a large, well-understood database. Rugby hohlraums offer the potential for added absorbed x-ray energy and, in turn, increased margin to hohlraum flux asymmetry, mix and target fabrication errors.

An immediate hurdle in arguing for the viability of double-shell ignition is delivery of an ignition-ready prototype target. The current designs call for nanoscience-based materials properties that ameliorate hydrodynamic instability and offer room temperature fielding. Many of these required properties have now been demonstrated, including nano-crystalline $\mathrm{Au}-\mathrm{Cu}$ alloys, nano-porous metallic foams, and nano-crystalline ablator shells. A technology demonstration prototype of an ignition double shell is scheduled for 2008.

\section{Acknowledgments}

We gratefully acknowledge the collaboration with the Fraunhofer Institute for Applied Solid State Physics on developing the diamond capsules; Juergen Biener, for his scientific contributions and liaison role in the diamond collaboration; Alan Jankowski, for delivering the nano-crystalline $\mathrm{Au} / \mathrm{Cu}$ inner-shell samples; Grey Nyce, John Poco and Joe Satcher for producing the ultra-low-density copper foams; Oggie Jones for useful discussions on recent Omega experiments with phase plates; and Nino Landen for useful input. This work was

performed under the auspices of the U.S. Department of Energy by the University of California Lawrence Livermore National Laboratory under Contract No. W-7405-Eng-48.

\section{Figure captions}


Fig. 1: Schematic of $2 \mathrm{MJ}$ (left) and $1 \mathrm{MJ}$ double-shell target designs for the NIF driven at $3 \omega$. Note the bimetallic composition of the inner shell and the material match $(\mathrm{Cu})$ between the outside of the inner shell and the metallic foam for hydrodynamic instability control.

Fig. 2a-b: (a) Schematic of 2 MJ rugby-hohlraum design showing laser cones and dimensions; (b) geometrical construction for a rugby hohlraum.

Fig. 3: Normalized second Legendre coefficient of outer-shell ablation pressure fluence near time of shell collision for $2 \mathrm{MJ}$ rugby hohlraum design (red; see Fig. 1) and cylindrical hohlraum (blue) for equal wall surface area versus outer-shell scale factor. The rugby (cylinder) LEH is $41.6 \%$ (48\%), and the cylinder hohlraum length (diameter) is $1.3 \mathrm{~cm}(0.748 \mathrm{~cm})$.

Fig. 4: Total power history (heavy solid line) for 2 MJ rugby hohlraum design with inner (dashed) and outer cone (solid) contributions.

Fig. 5: Normalized second and fourth Legendre coefficient of outer shell ablation pressure versus time for $2 \mathrm{MJ}$ rugby hohlraum design.

Fig. 6: (left) SRS (red) and SBS (blue) streaks for $2 \mathrm{MJ}$ rugby hohlraum design with $1.5 \mathrm{~mm}$ diameter focal spots; (right) tabulated peak SRS and SBS gain-length products versus cone angle. 
Fig. 7: Material (left) and density (right) fill plot of $1 \mathrm{MJ}$ rugby-hohlraum design (See Fig. 1) at time of peak neutron production.

Fig. 8: Tabulated peak SRS and SBS gain-length products versus cone angle for 1 MJ rugbyhohlraum design.

Fig. 9: Transmission electron micrograph of planar electrodeposited $\mathrm{Au}-\mathrm{Cu}$ sample.

Fig. 10: $1 \mathrm{~mm}$-scale electrodeposited $\mathrm{Au}-\mathrm{Cu}$ shells over glass mandrels. Inner Au layer is $5 \mu \mathrm{m}$ thick; $\mathrm{Cu}$ overcoat is $5 \mu \mathrm{m}$.

Fig. 11a-b: (a) $85 \mathrm{mg} / \mathrm{cc} \mathrm{Cu}$ foam with $12 \mathrm{wt} \% \mathrm{SiO}_{2}$ aerogel matrix; (b) $50 \mathrm{mg} / \mathrm{cc} \mathrm{CuO}$ foam with sub-micron pore sizes.

Fig. 12: Simulated Atwood number $\left[\left(\rho_{2}-\rho_{1}\right) /\left(\rho_{1}+\rho_{2}\right)\right]$ for outer-shell/foam interface versus time for $\mathrm{Cu}+$ aerogel foam (green), pure $\mathrm{Cu}$ foam (blue) and pure aerogel (red). Onset of RayleighTaylor instability growth is at $5 \mathrm{~ns}$.

Fig. 13: Optical image of $2 \mathrm{~mm}$ diameter, $90 \mu \mathrm{m}$ thick, nano-crystalline, polished diamond shells.

\section{References}


[1] J.A. Paisner, E.M. Campbell and W.H. Hogan, Fusion Technol. 26, 755 (1994).

[2] J.D. Lindl, P.A. Amendt, R.L. Berger et al., Phys. Plasmas 11, 339 (2004).

[3] E.M. Campbell et al., Laser Part. Beams 9, 209 (1991).

[4] T.R. Boehly et al., Opt. Commun. 133, 495 (1997).

[5] E.L. Dewald, O.L. Landen, L.J. Suter et al., Phys. Plasmas 13, 056315 (2006).

[6] J.D. Lindl, Inertial Confinement Fusion (Springer-Verlag, New York, 1998).

[7] P. Amendt, J.D. Colvin, J.D. Ramshaw et al., Phys. Plasmas 9, 2221 (2002).

[8] M.N. Chizhkov, N.G. Karlykhanov, V.A. Lykov et al., Laser Part. Beams 23, 261 (2005).

[9] L.J. Suter, A.A. Hauer, L.V. Powers et al., Phys. Rev. Lett. 73, 2328 (1994).

[10] S.W. Haan, M.C. Herrmann, T.R. Dittrich et al., Phys. Plasmas 12, 056316 (2005).

[11] P.A. Amendt, H.F. Robey, H.-S. Park et al., Phys. Rev. Lett. 94, 065004 (2005).

[12] R. Hibbard et al., Fusion Technol. 45, 117 (2005).

[13] J.L. Milovich, P. Amendt, M.M. Marinak et al., Phys. Plasmas 11, 1552 (2004).

[14] P. Amendt, Phys. Plasmas 13, 042702 (2006).

[15] D.A. Callahan, P.A. Amendt, E.L. Dewald et al., Phys. Plasmas 13, 0560308 (2006).

[16] S.M. Pollaine, D.K. Bradley, O.L. Landen et al., Physics Plasmas 8, 2357 (2001).

[17] J.M. Wallace, T.J. Murphy, N.D. Delamater et al., Phys. Rev. Lett. 82, 3807 (1999).

[18] O.L. Landen, P.A. Amendt, L.J. Suter et al., Phys. Plasmas 6, 2137 (1999).

[19] O.S. Jones and J. Schein, this meeting (2006).

[20] L.V. Powers, R.L. Berger, R.L. Kauffman et al., Phys. Plasmas 2, 2473 (1995).

[21] E.A. Williams, Phys. Plasmas 13, 056310 (2006). 
[22] H.N. Kornblum, R.L. Kauffman and J.A. Smith, Rev. Sci. Instrum. 57, 2179 (1986).

[23] O.S. Jones and S. Regan (private communication, 2006).

[24] E.A. Williams (private communication, 2000)

[25] M.M. Marinak, G.D. Kerbel, N.A. Gentile et al., Phys. Plasmas 8, 2275 (2001).

[26] E.O. Hall, Proc. Phys. Soc. (London), B 64, 747 (1951); N.J. Petch, J. Iron Steel Inst. 174, 25 (1953). 
Fig. 1
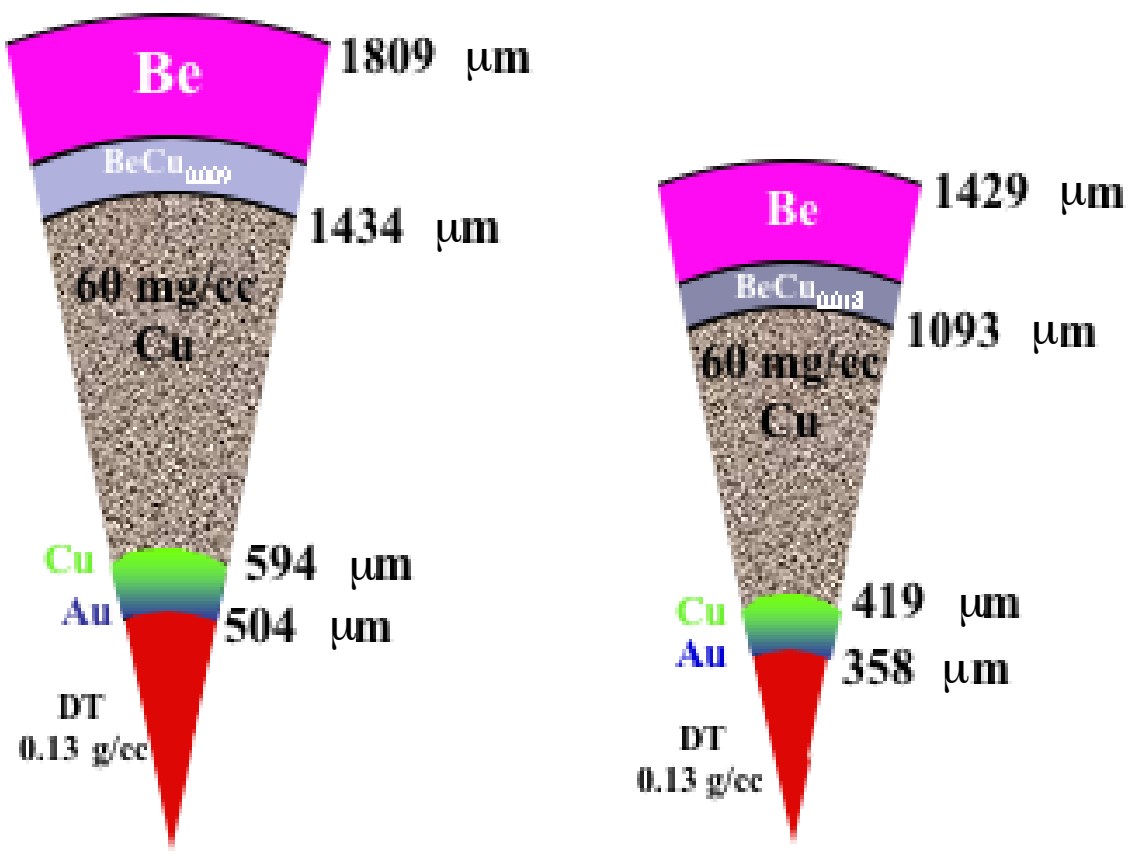

2 MJ

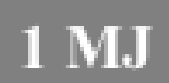


Fig. 2a

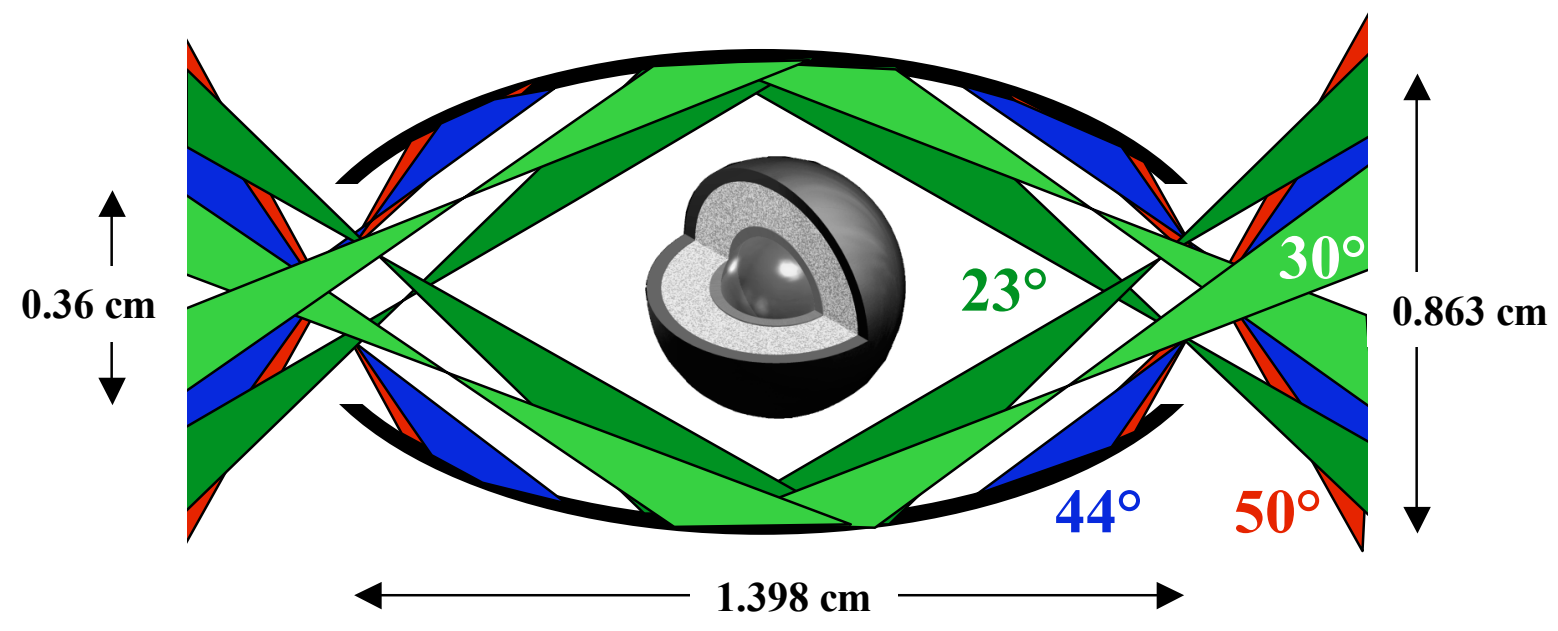


Fig. 2b

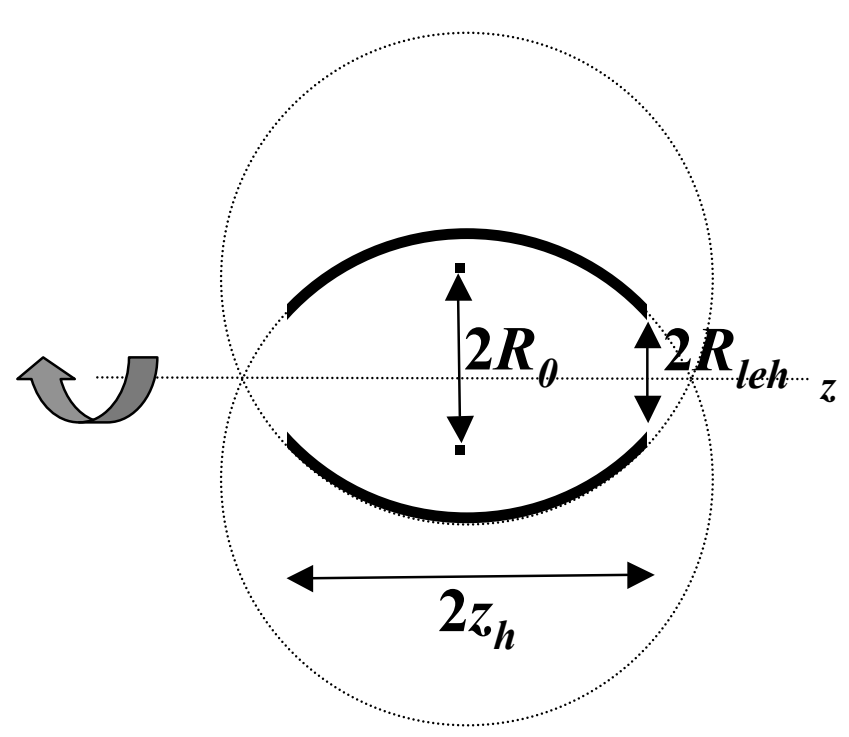


Fig. 3

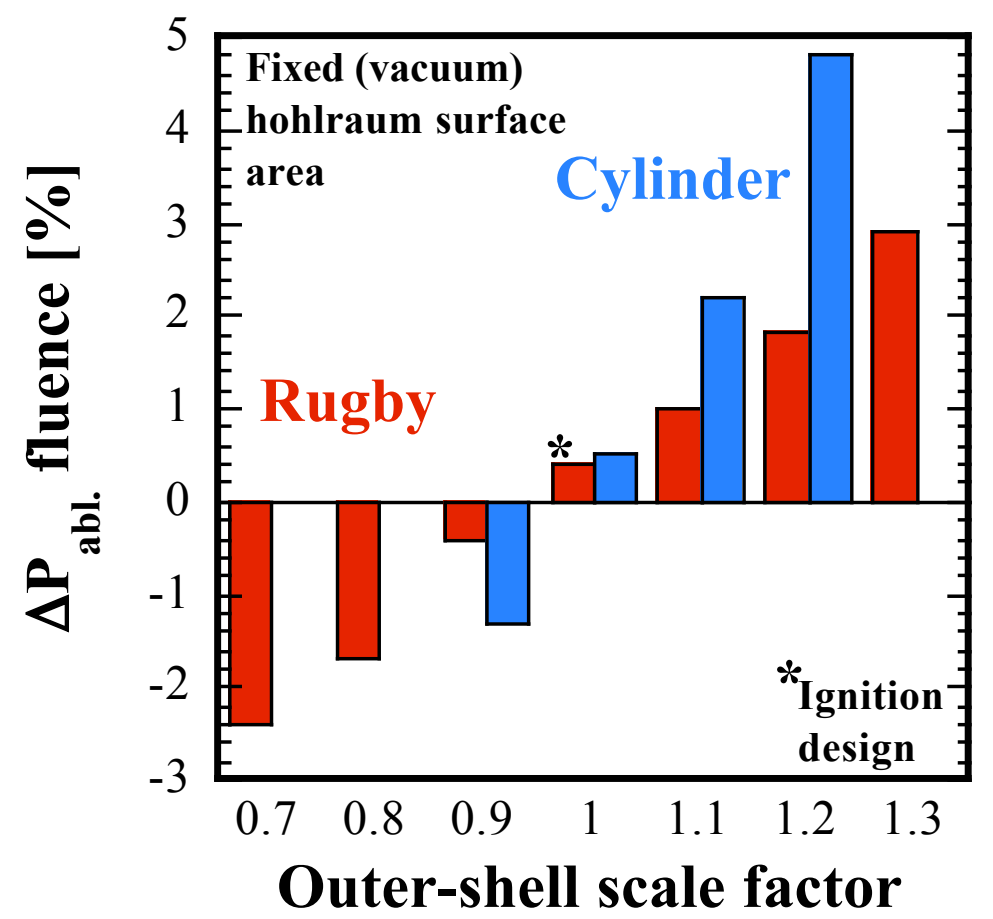


Fig. 4

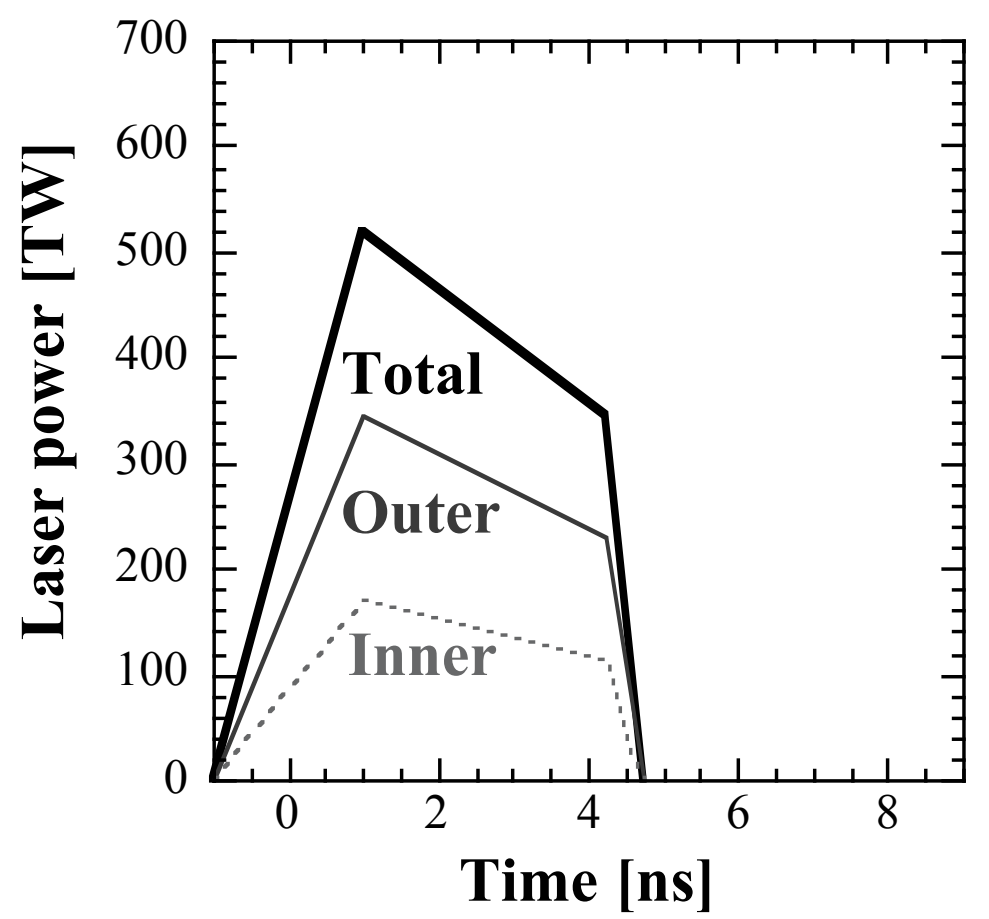


Fig. 5

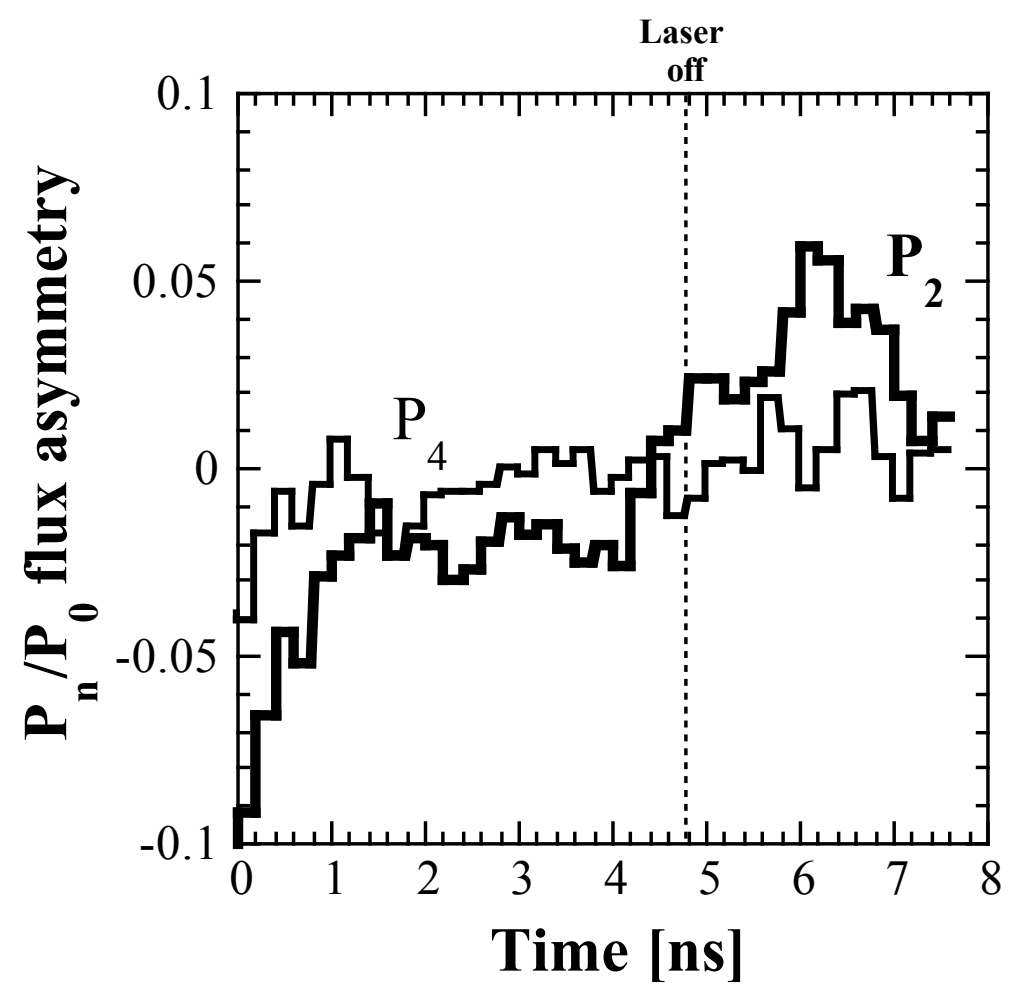


Fig. 6
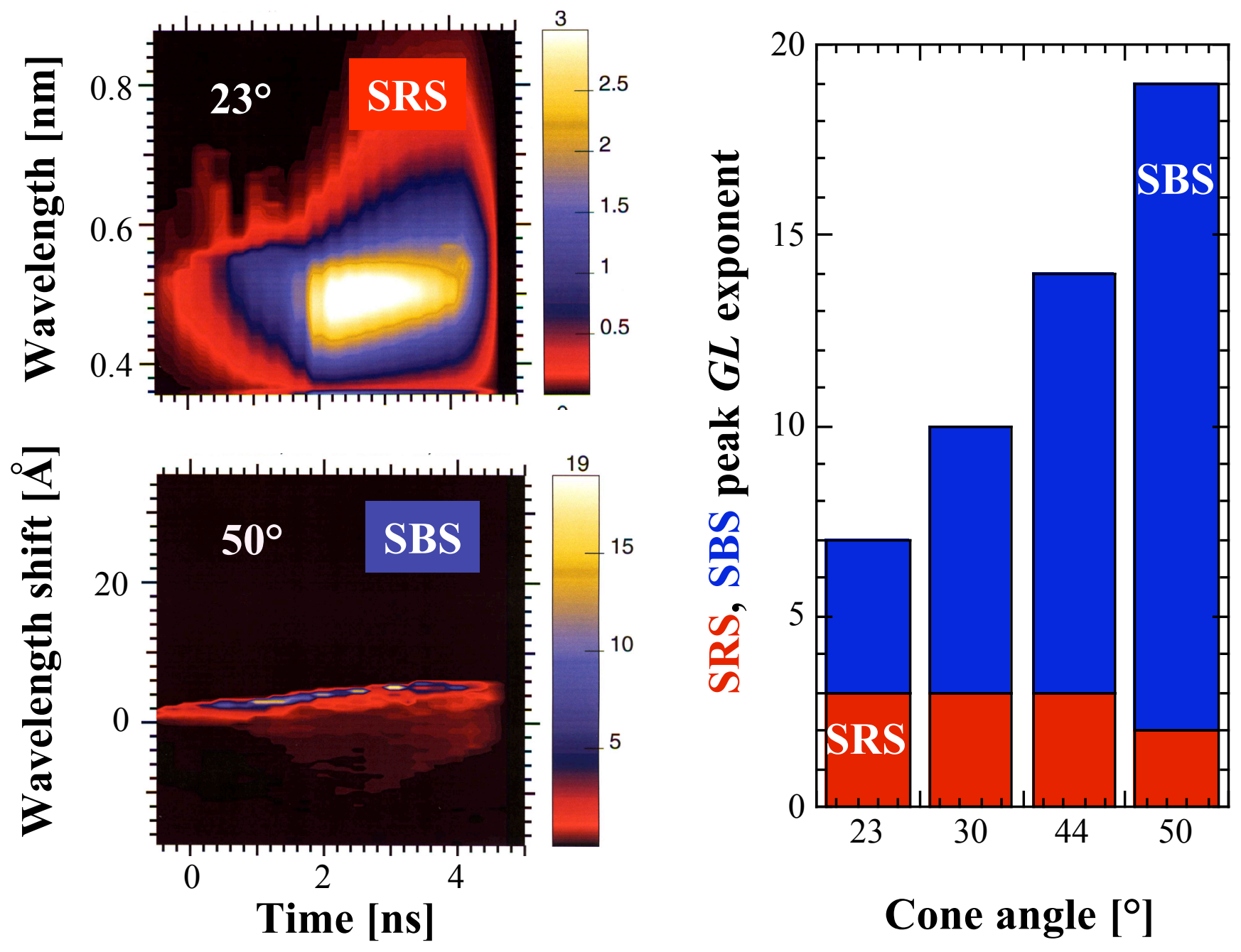
Fig. 7

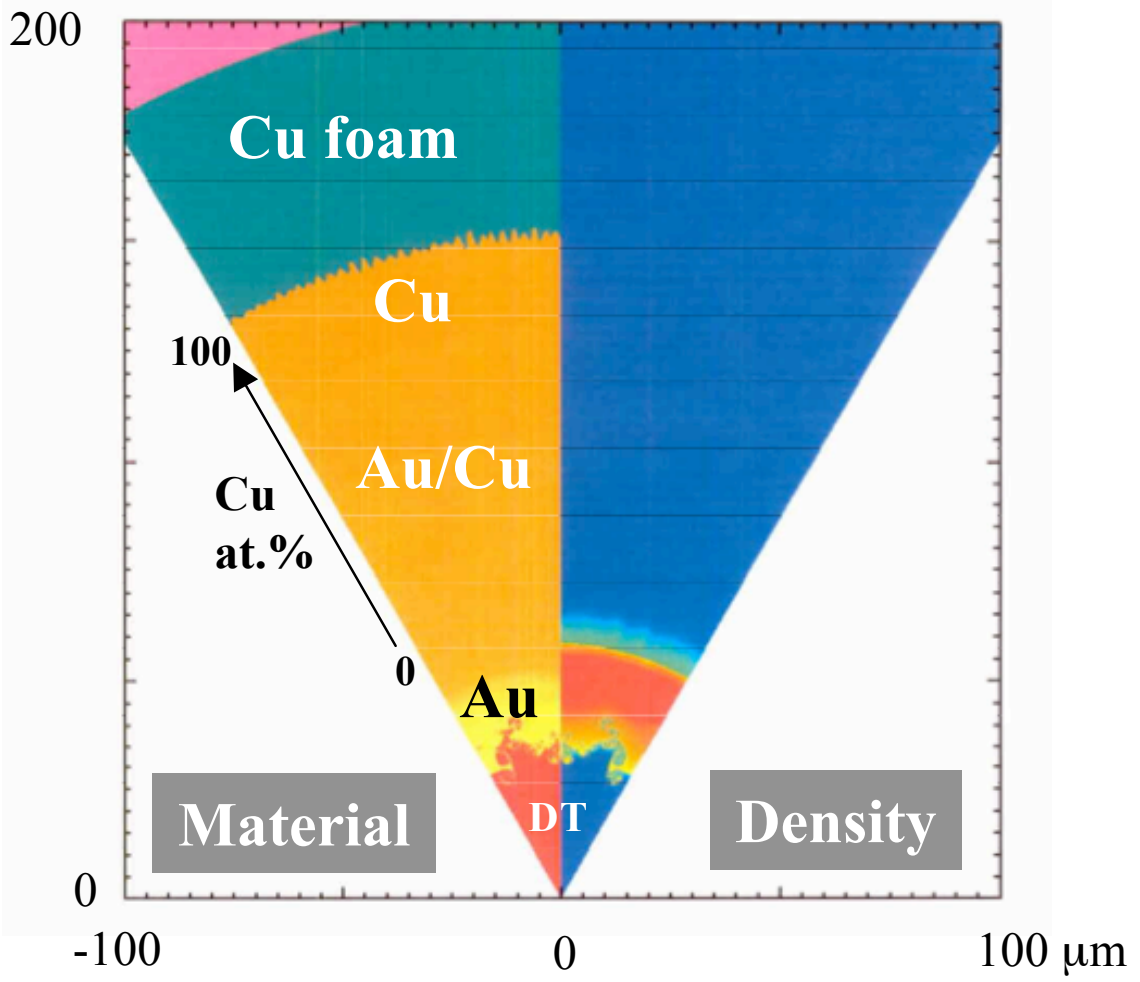


Fig. 8

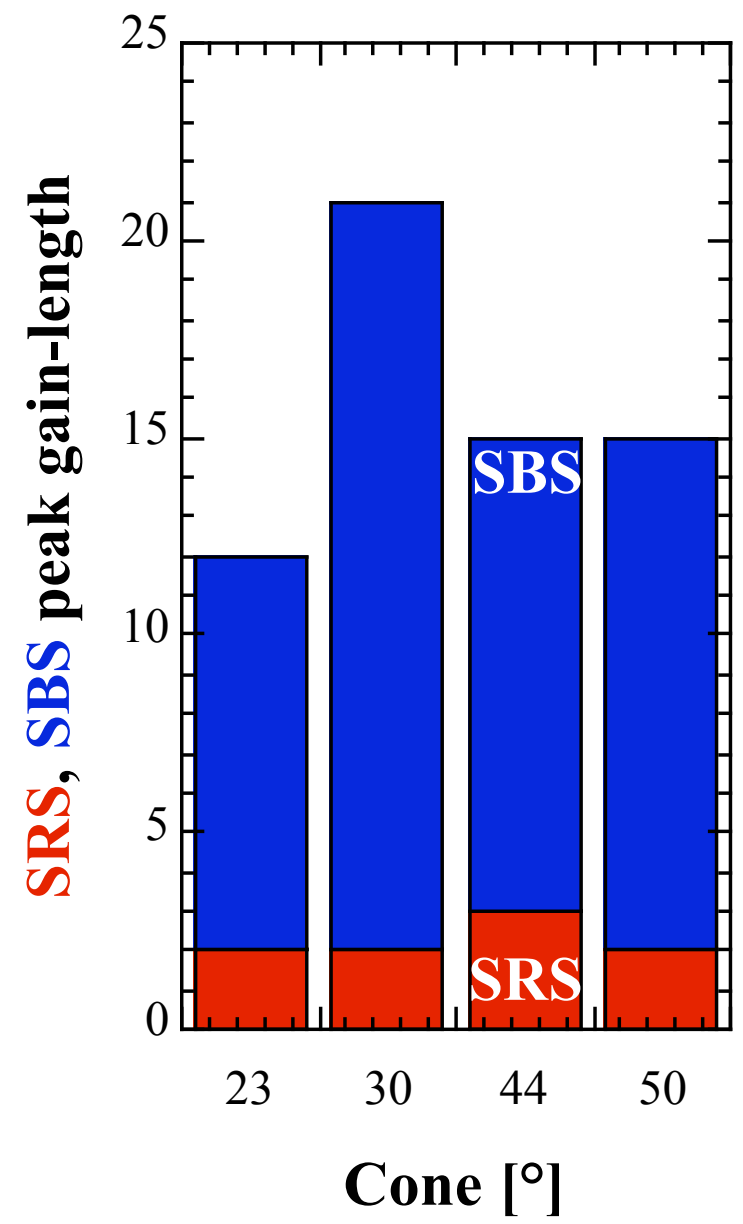


Fig. 9

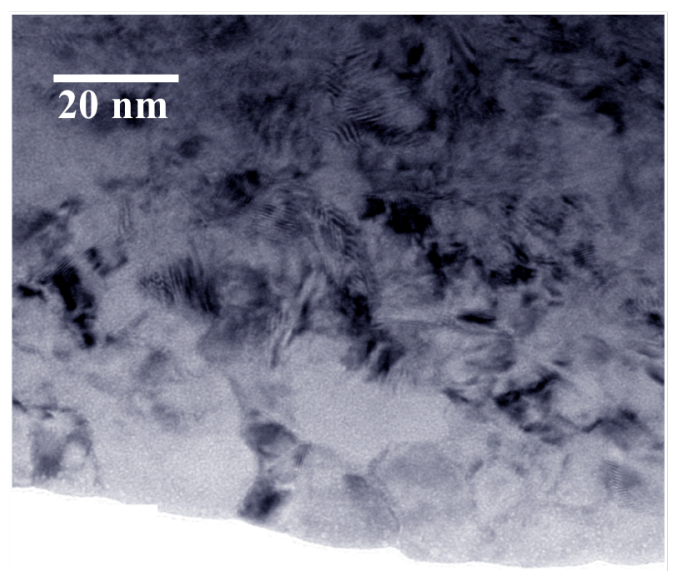


Fig. 10

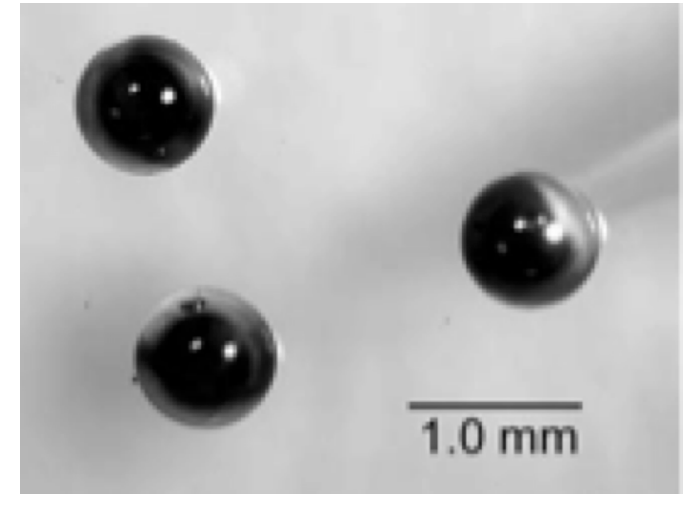


Fig. 11a-b

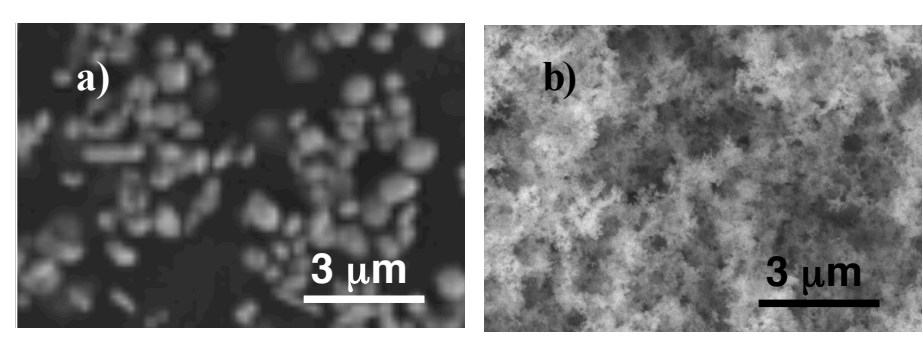


Fig. 12

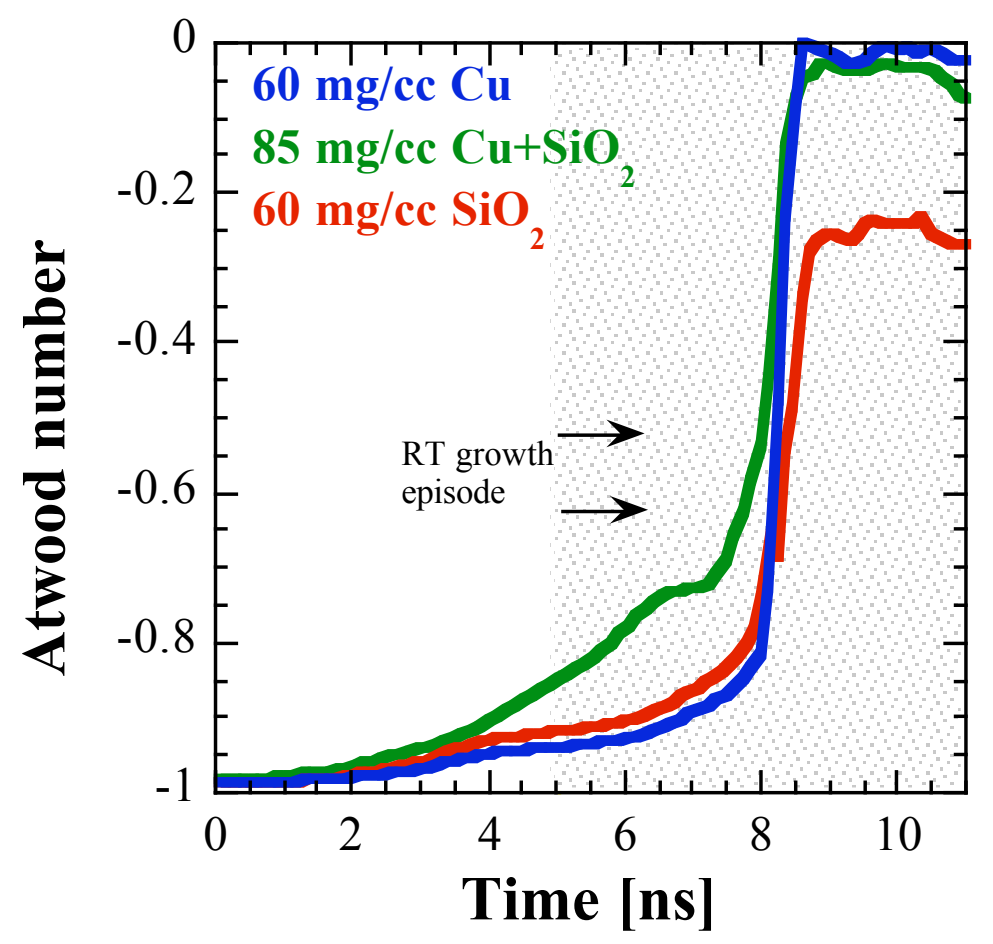


Fig. 13

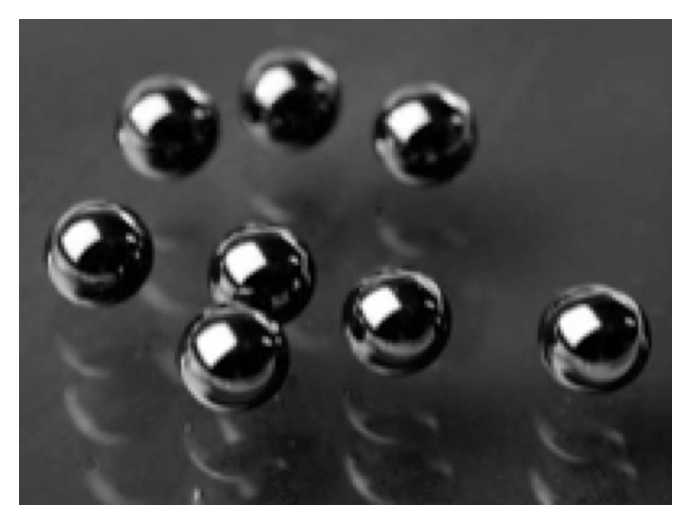

